\title{
THE CAPTOPRIL-DIGOXIN MULTICENTER RESEARCH GROUP STUDY ON THE COMPARATIVE STEPS OF CAPTOPRIL AND DIGOXIN IN PATIENTS WITH MILD- MODERATE HEART FAILURE: IMPLICATIONS FOR THERAPY
}

\begin{abstract}
SUMMARY. It is argued that the basis of therapy in a patient with mild-to-moderate congestive heart failure should be a combination of a diuretic and a converting enzyme inhibitor, with the further addition of digoxin if necessary.
\end{abstract}

KEY WORDS. captopril, digoxin, congestive heart failure

The therapy of patients with mild-moderate heart failure has changed relatively little over the past several decades. Although vasodilators and, in particular, the converting enzyme inhibitors have been shown to be of value in improving exercise performance and survival in patients with severe heart failure [1-3], they have not found use in the patient with mildmoderate heart failure. Most patients with mildmoderate heart failure, at least in the United States of America, are treated with a diuretic alone or with the combination of a diuretic and digoxin [4]. The recent study on the comparative effect of captopril and digoxin in patients with mild-moderate heart failure [5] is the first major study to address the issue of the efficacy of both of these agents.

In brief, patient inclusion criteria were age less than 75 years, sinus rhythm, left ventricular ejection fraction of $\leqslant 40 \%$, and a treadmill exercise performance less than that predicted for the age and sex, but greater than 4 minutes on a modified Naughton protocol, and not limited by angina pectoris after withdrawal of baseline digoxin and/or vasodilators. Heart failure in patients solicited for this study was due to either ischemic heart disease, primary myocardial disease, or to patients remaining in heart failure without significant valvular regurgitation after valve replacement.

Four hundred and sixty-four patients met the entry criteria and entered the stabilization period after withdrawal from digoxin or vasodilators, and were maintained on a stable dose of a diuretic if needed. Less than $10 \%$ of the patients withdrawn from digoxin and/or vasodilators showed any cardiac deterioration, such as progressive heart failure, atrial fibrillation, or death during the stabilization period. Three hundred

\author{
Bertram Pitt, Sidney Goldstein \\ University of Michigan Medical Center, Ann Arbor, Michigan, \\ USA
}

patients met the entry criteria and were randomized to one of three treatment strategies: captopril $50 \mathrm{mg}$ t.i.d., digoxin $0.25 \mathrm{mg}$ q.d., adjusted to a therapeutic level by serum digoxin determination, or to placebo. All patients were maintained on a diuretic if needed. Once in the study, diuretic dosage could be adjusted according to patient symptoms. Of the 300 patients who were randomized into the double-blind study, $83 \%$ were males, the mean age was 57 years, the mean left ventricular ejection fraction was $25 \%$, and the mean exercise time on a modified Naughton protocol was 560 seconds. Two thirds of the patients had ischemic heart disease and one third had primary myocardial disease. The patients were followed with repeat exercise testing, radioisotope ejection fraction, Holter ECG recording, and a clinical evaluation for 6 months or until, because of symptoms of progressive heart failure, openlabel therapy with a vasodilator and/or digoxin was instituted.

One of the most important findings in this study was that patients assigned to the placebo group and maintained on a diuretic alone had significantly more episodes of manifest heart failure, as evidenced by visits to the emergency room or hospitalization for heart failure, compared to either the digoxin- or captopril-treated groups. This increased incidence of progressive heart failure occurred despite the fact that the patients assigned to the placebo group had a significantly greater increase in diuretic usage over the follow-up period. These data suggest that maintenance

Addiress for correspondence and reprint requests: Bertram Pitt, MD, Division of Cardiology, University of Michigan Medical Center, Ann Arbor, MI 48109-0366, USA 
of a patient with mild-moderate heart failure on a diuretic alone is not adequate therapy. Another important result was the finding that the patients assigned to the captopril group and maintained on a diuretic as needed had a significantly greater increase in exercise performance and improvement in functional class than the placebo group, while the digoxin group did not. It should, however, be pointed out that the difference in exercise performance and functional improvement between captopril and digoxin was not statistically significant. There was, however, a significant reduction in ventricular ectopic activity in the captopril group compared to the digoxin group in patients with at least 10 ventricular ectopic beats per hour on their baseline Holter ECG. In contrast to these results, the patients assigned to the digoxin group had a significant increase in their left ventricular ejection fraction compared to both the captopril and placebo groups. It should, however, be emphasized that in this and previous studies [6], there is no significant correlation between improvement in left ventricular ejection fraction and improvement in exercise performance or patient well-being. In regards to safety, there was a significant increase in hypotension in the captopril group, but these episodes were transient, were usually treated by decreasing diuretic dosage, and did not result in an increased drop-out rate compared to the digoxin group. The mortality rate was calculated at $10.5 \%$ per year but the power of the study was insufficient to make any comments regarding the relative effects of the treatment strategies on survival.

These results and the increasing evidence suggesting the beneficial effects of vasodilators and, in particular, the converting enzyme inhibitors, suggests consideration of a new strategy for the treatment of patients with mild-moderate heart failure. We propose that patients with mild-moderate heart failure should be treated and maintained with a combination of a diuretic and a converting enzyme inhibitor. As pointed out above, maintenance on a diuretic alone is inadequate to prevent progressive heart failure. Diuretics alone tend to stimulate secondary neurohumoral mechanisms, including catecholamines, vasopressin, and the renin-angiotesin system [7,8]. Diureticinduced activation of the renin-angiotensin system results in peripheral as well as coronary vasoconstriction [9]. Secondly, diuretics when given alone tend to cause hypokalemia and hypomagnesemia, which may predispose patients to ventricular arrhythmias and sudden cardiac death [10]. When a diuretic is combined with a converting enzyme inhibitor, the secondary nuerohumoral activation induced by diuretics is blunted and the peripheral and coronary vasoconstric- tion is relieved [9]. Similarly, diuretic-induced hypokalemia and hypomagnesemia is prevented by converting enzyme inhibition.

Although the combination of a converting enzyme inhibitor and a diuretic resulted in a better improvement in exercise performance and functional class than digoxin and a diuretic in the multicenter study [4], this difference, as pointed out above, is not significant. It could, however, be argued that the study design was biased against digoxin, since in order to enter the random trial, the patient had to tolerate withdrawal of digoxin and, in fact, approximately $7.5 \%$ of patients entering the washout period demonstrated a need for digoxin and were not randomized. If these patients were included, it is possible that the combination of digoxin and a diuretic might be as efficacious as the combination of a converting enzyme and a diuretic in regard to exercise performance and functional class.

There appears to be little doubt that digoxin is effective in patients with severe heart failure, especially those characterized by a dilated left ventricle and the presence of a third heart sound [11]. Its role in the patient with mild-moderate heart failure is, however, questionable, Several previous studies, including the recent German-Austrian study of xamoterol in over 300 patients, have failed to show a significant effect of digoxin on exercise performance [12]. Even if one grants a modest effect on exercise performance and patient well-being in patients with mild-moderate heart failure receiving digoxin equal to or greater than that seen with captopril and a diuretic, this has to be weighed against the potential risk of digitalis toxicity, especially in elderly patients, and the uncertain effect of digoxin on survival. Several authors have suggested that digoxin might have an adverse effect on survival in patients with ischemic heart failure. The studies upon which this view is based are, however, retrospective and, as pointed out by Yusuf et al. [13], until a prospective random trial of digoxin in which mortality as the endpoint is carried out, no definitive conclusions in regard to the risk of digoxin can be reached. However, recent animal studies by Lynch et al. [14] in which conscious dogs with an anterior myocardial infarction were randomized to digoxin adjusted to the therapeutic range or placebo, and were then subjected to ischemia of the left circumflex coronary arterial bed, showed an increased mortality in animals assigned to the digoxin group. This study suggests caution in the use of digoxin in ischemic heart failure. While it is hazardous to extrapolate from any animal model to the more complex situation in patients with heart failure, it is fair to demand that there be a clear benefit to the use of digoxin 
before one accepts even the theoretic risk of increased mortality. Given the available evidence, it is difficult to recmommend digoxin and a diuretic as the sole therapy in the patient with mild-moderate heart failure. It should, however, be emphasized that if the patient with mild-moderate heart failure treated with a converting enzyme inhibitor and a diuretic remains symptomatic, then digoxin should not be withheld and may be of significant benefit. Similarly, in the patient who cannot tolerate a converting enzyme inhibitor or vasodilation with isorbide dinitrate and hydralazine, a combination that has been shown to be effective in the improvement of survival of patients with heart failure [1], digoxin is indicated. One could argue for the use of a diuretic, converting enzyme inhibitor, and digoxin in all patients with mild-moderate heart failure. If, however, the patient on a diuretic and converting enzyme inhibition is asymptomatic or relatively so, there would seem to be little reason to add digoxin in patients with underlying ischemic heart disease until there is further information on its risk.

The strategy for the therapy of patients with mildmoderate heart failure outlined above is in part based upon the assumption that the beneficial effects of converting enzyme inhibition seen in patients with severe heart failure [2] will also be evident in the patient with mild-moderate heart failure. Whether this assumption is valid will depend in part upon the results of several ongoing large prospective trials, including the study of left ventricular dysfunction (SOLVD) and the comparison of the effect of converting enzyme inhibition to the combination of isorbide dinitrate and hydralazine on mortality (VHEFT). Until the results of these and other ongoing studies are available, it seems reasonable, based upon the evidence presented above, to recommend that the basis of therapy in the patient with mild-moderate heart failure be a combination of a diuretic and a converting enzyme inhibitor.

\section{References}

1. Cohn JN, Archibald DG, Ziesche S, et al. Effect of vasodilator therapy on mortality in chronic congestive heart failure. $N$ Engl $J$ Med 1986;314:1547-155 2.

2. The CONSENSUS Trial Study Group. Effects of enalapril on mortality in severe congestive heart failure. $N$ Engl J Med 1987;316:1429-1435.

3. Captopril Multicenter Study Group. A placebo trial of captopril in refractory congestive heart failure. J Am Coll Cardiol 1983;2:755-763.

4. Hlatky MA, Fleg JL, Hinton PC, et al. Physician practice in the management of congestive heart failure. $J \mathrm{Am}$ Coll $\mathrm{Car}$ diol 1986;8:966-970.

5. The Captopril-Digoxin Multicenter Research Group. Comparative effects of therapy with captopril and digoxin in patients with mild to moderate heart failure. JAMA 1988;259:539-544.

6. Franciosa JA, Park M, Levine TB. Lack of correlation between exercise capacity and indexes of left ventricular performance in heart failure. Am J Cardiol 1981;47:33-39.

7. Ikram H, Chan W, Espiner EA, Nicholls MG, et al. Hemodynamic and hormone response to acute and chronic furosemide therapy in congestive heart failure. Clin Sci 1980;59:443-449.

8. Bayliss J, Norell M, Canepa-Anson R, et al. Untreated heart failure: Clinical and neuronedocrine effects of introducing diuretics. Br Heart $J$ 1987;57:17-22.

9. Magrini F, Shimizu M, Roberts N, et al. Converting-enzyme inhibition and coronary blood flow. Circulation 1987; 75(Supp I):168-174.

10. Johansson BW. Hypokalemia in cardiac decompensation. Acta Pharmocol Toxicol 1984;54(Supp I):103-106.

11. Lee DC, Johnson RA, Bingham JE, et al. Heart failure in outpatients: A randomized trial of digoxin versus placebo. $N$ Engl J Med 1986;306:699-705.

12. The German-Austrian Xamoterol Study Group. Doubleblind placebo-controlled comparison of digoxin and xamoterol in chronic heart failure. Lancet 1988;1:489-493.

13. Yusuf S, Wittes J, Bailey K, et al. Digitalis-a new controversy regarding an old drug: The pitfalls of inappropriate methods. Circulation 1986;73:14-18.

14. Lynch JJ, Montgomery DG, Lucchesi BR. Facilitation of lethal ventricular arrhythmias by therapuetic digoxin in conscious post-infarction dogs. Am Heart $J$ 1986;111:883-890. 